\title{
Flexible grippers for industrial robots - comparison of features of low-cost 3D printed component
}

\author{
Piotr Zbroja ${ }^{1}$, Ksawery Szykiedans ${ }^{1, *}$, and Wojciech Credo ${ }^{1}$ \\ ${ }^{1}$ Warsaw University Of Technology, Faculty of Mechatronics, 02-525 Warsaw, ul. św. A. Boboli 8, \\ Poland
}

\begin{abstract}
The aim of presented work was to analyse the feasibility of using 3D-print technology in robotics based on the production of industrial robot flexible grippers. For selected geometry of gripper single finger available 3D printing techniques has been analysed. The study made by authors uses the following additive technologies and devices: SLS (Selective laser Sintering) and FDM (Fused deposition modelling). As a prior an analyses of capabilities of individual technologies were done by testing the quality of the 3D CAD model recreated on test print-outs. Based on the printed gripper, its functionality, and strength properties were examined. Strength of grapplers was tested with a use of an MTS test machine under repeating deflexion simulating standard operational cycle of a gripper. Test proved that at least few thousands of cycle are possible to be made by a $3 \mathrm{D}$ printed gripper. What interesting gripper made with use of the less advanced printer showed different wear behaviour than an one made on the more advanced. First one showed almost instantaneous start of slow and constant strength degradation while the second one proved to have a stable deflexional capability by almost twice an number of cycles. More isotropic structure of an SLS printed gripper caused the best results of all tested ones.
\end{abstract}

Keywords: Flexible robotic grippers, additive manufacturing, fatigue test, fused deposition modelling, selective laser sintering, layer plastic deposition

\section{Introduction}

During following years, we can see the ever wider use of 3D printing in prototype solutions for the robotics industry and beyond. Grippers and their components made in additive techniques are already used not only in amateur solutions, but are also offered by large companies involved in the robotics industry. With the use of additive technology, we can provide a unique solution for your application in a very short time by optimizing the cost, mass, and dimension of individual elements. A very important aspect that causes implementation of a given solution is the fatigue strength of its components. In the case of dynamic loads or deformations that change over time (e.g. periodically), the destruction of the polymer sample may occur at medium stress or deflection by up to $80 \%$ less than under static conditions, if only the varying stress or deflection works long enough.

${ }^{*}$ Corresponding author: ksawery.szykiedans@pw.edu.pl

Reviewers: Darina Ondrušová, Krzysztof Talaśka 
Flexible grippers have been developed for many years [1-3], but their development despite many advantages has been limited by the limitations in manufacturing. The development of $3 \mathrm{~d}$ printing methods has facilitated the execution of complex spatial structures with adequately low stiffness for use in flexible grippers. One of the examples of such grapples the authors devoted their attention..

\subsection{Festo DHDG gripper $[5,6]$}

The grippers of DHDG series are the most innovative handhelds from FESTO®. They use so called Fin Ray Effect ${ }^{\circledR}$, a behaviour inspired on a fish fin movements. Whole gripper consists of three fingers and a handle coupled to a pneumatic system. This grapple, thanks to its bionic manner of capture, enables safe, yet secure, manipulation of light, delicate (in terms of vulnerability to damage), brittle objects, even having very different shapes [4]. The main advantage of those grippers is their optimisation of the structure by the use of double-acting pneumatic system and interchangeable fingers. Both the handle and the gripper finger are made of sintered powder of polyamide in the SLS technology (Selective Laser Sintering).

This type of gripper can be used in applications where it is required to manipulate irregularly shaped objects whose initial position in the manufacturing process changes. With the ability of providing a minimum pressure on the gripping surfaces to achieve the effect of a gripper finger adjustment, these grippers work very well with very delicate objects. Therefore, quite quickly after the product announcement by FESTO, there have been attempts to use this idea with both the use of some stock subcomponents [7] and with the use of additive manufacturing techniques [8]. For these reasons, it was decided to conduct a study on the possibility of performing similar grapples available with rapid prototyping methods. Our own model of such type gripper have been developed for further tests (Fig.1).

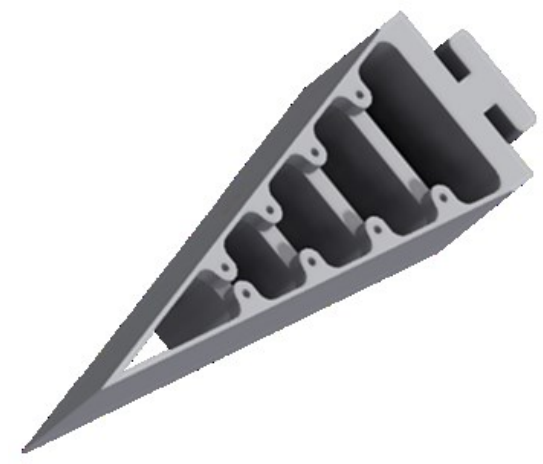

Fig. 1. Flexible finger for a robotic gripper. The form designed by P. Zbroja

\section{Workflow}

Assumed work was divided into three stages:

Stage 1

- gripper finger design made in Autodesk Inventor software, possible to perform in each of the additive technologies described,

- simulation of stress analysis of the designed gripper finger in Autodesk Inventor software to determine the stresses occurring in the assembly and to introduce possible corrections to the final model before the final production in each technologies

Stage 2 
- manufacturing of gripper finger components and quality measurements of 3D CAD model in particular additive technologies,

- demonstration of main differences between each additive technologies and the impact of events occurring during the manufacturing process and after its completion on the final quality of 3D CAD model reproduction as well as its strength properties.

Stage 3

- performance of fatigue strength test by cyclical loading of adaptive gripper fingers made in each additive technologies.

\section{Specimens}

\subsection{D model preparation and validation}

The aim of the experiment was to explore the possibilities of low-budget incremental technologies (LPD) in the implementation of the adaptive gripper finger solution and to summarize them with professional incremental technologies (FDM and SLS). The adaptive finger of the gripper, made up of sub-components proper for all technologies used for research (FDM, SLS, LPD), has been designed to achieve the test objective. Designed form was only based on DHGG gripper it has been optimized to reduce stresses and to ensure manufacturability.

The gripper finger virtual model was subjected to a load of $13 \mathrm{~N}$ for the $\mathrm{x}$-axis displacement. The maximum displacement in the $\mathrm{x}$-axis of the geometric model under load was $9.45 \mathrm{~mm}$. The manner of gripper deflection under the load in the simulation coincides with its deformation in real.

Fig. 2 shows the results of the safety factor analysis for the designed geometric model of the gripper finger. Several sensitive areas (marked in black) are exposed to damage under load. On the basis of the simulation, it can be assumed that under the influence of cyclic load the previously defined force of the test object can be destroyed in places where the safety factor is lowest. Based on the results of the safety factor, the design of the underlying gripper

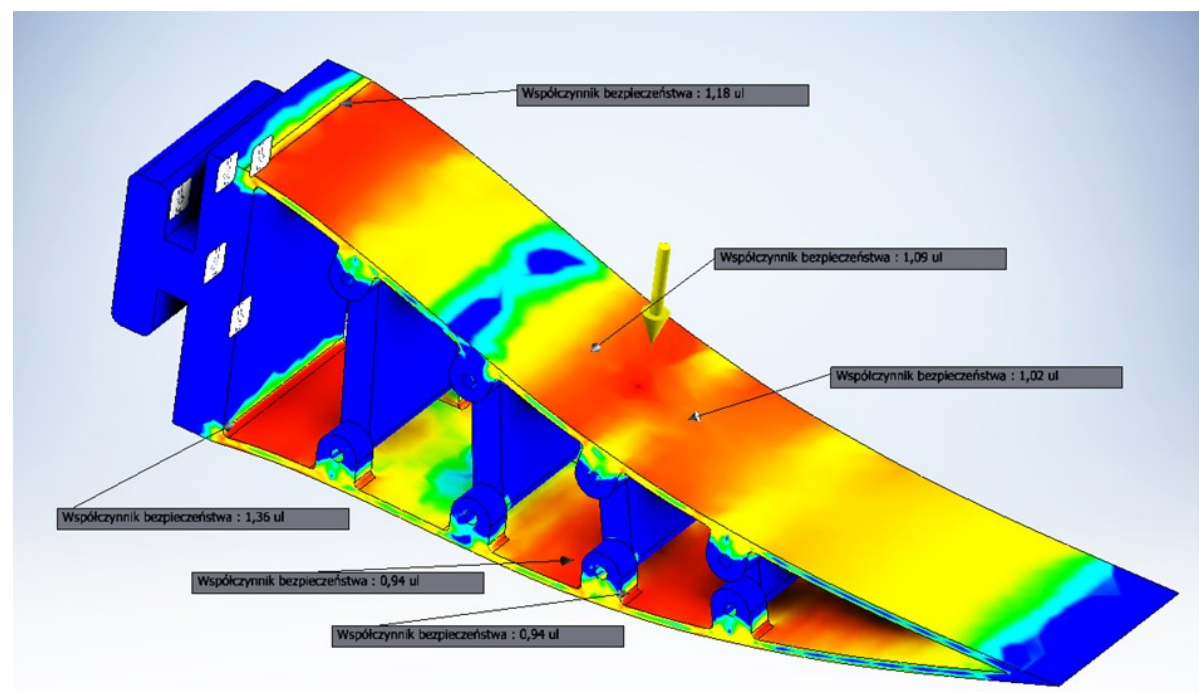

structure was changed to eliminate the possibility of gripping fractures due to the aggregate of stresses in defined, based on simulations, locations.

Fig. 2. Safety factor FEM analysis of a designed flexible finger 
In areas where the safety factor was the lowest design was reinforced, while maintaining the functionality of the gripper's finger, by increasing the rounding to $2.3 \mathrm{~mm}$.

\subsection{Specimens manufacturing and quality check}

Prepared virtual model, created with a use of Autodesk Inventor software, has been used to print specimens in the following additive techniques:

- LPD (Layer Plastic Deposition) - model made on the Zortax M200 printer,

- FDM (Fused Deposition Modeling) - model made on Dimension Elite printer,

- SLS (Selective Laser Sintering) - model made on a Formiga P100 printer.

Low-cost $3 \mathrm{~d}$ printing techniques are known for some manufacturing inaccuracies. There are deficiencies in the filling of the material (Fig. 3), uneven contraction or (which is essential for the manufacture of components such as the gripper) problems with printing thin walls. Therefore, after printing out the models, they were verified. The shape and dimension errors (Fig. 4) and no material fill have been looked for.

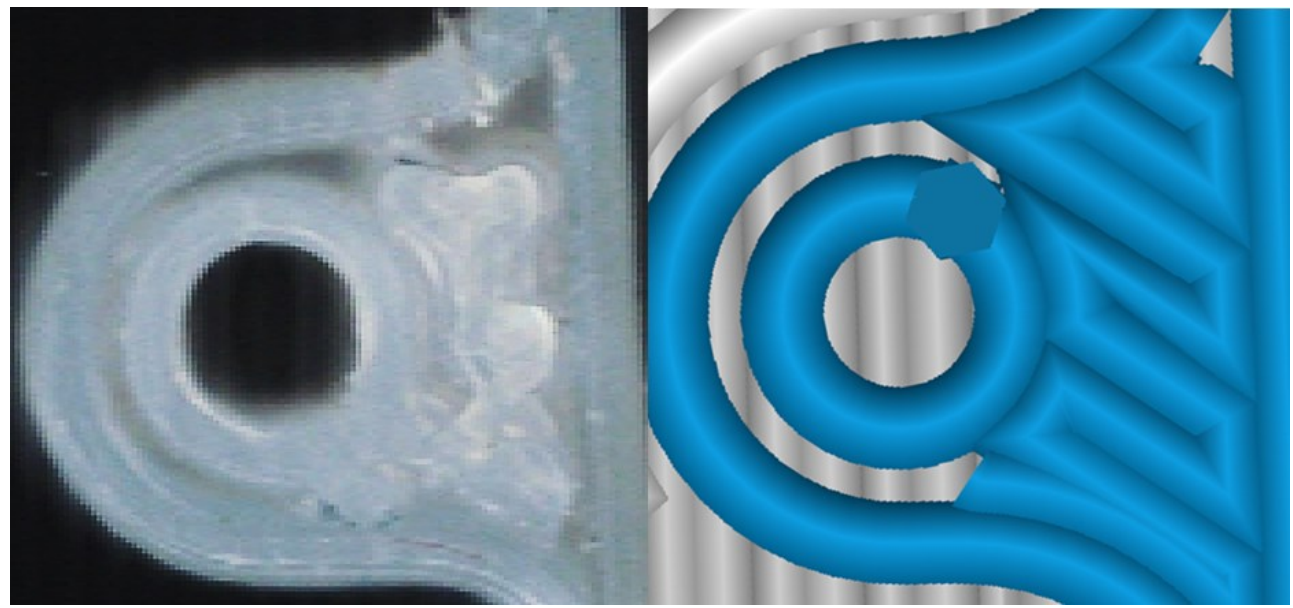

Fig. 3. Gaps caused by no material filling during manufacturing (left), issue has been earlier shown on simulation visualisation (right)

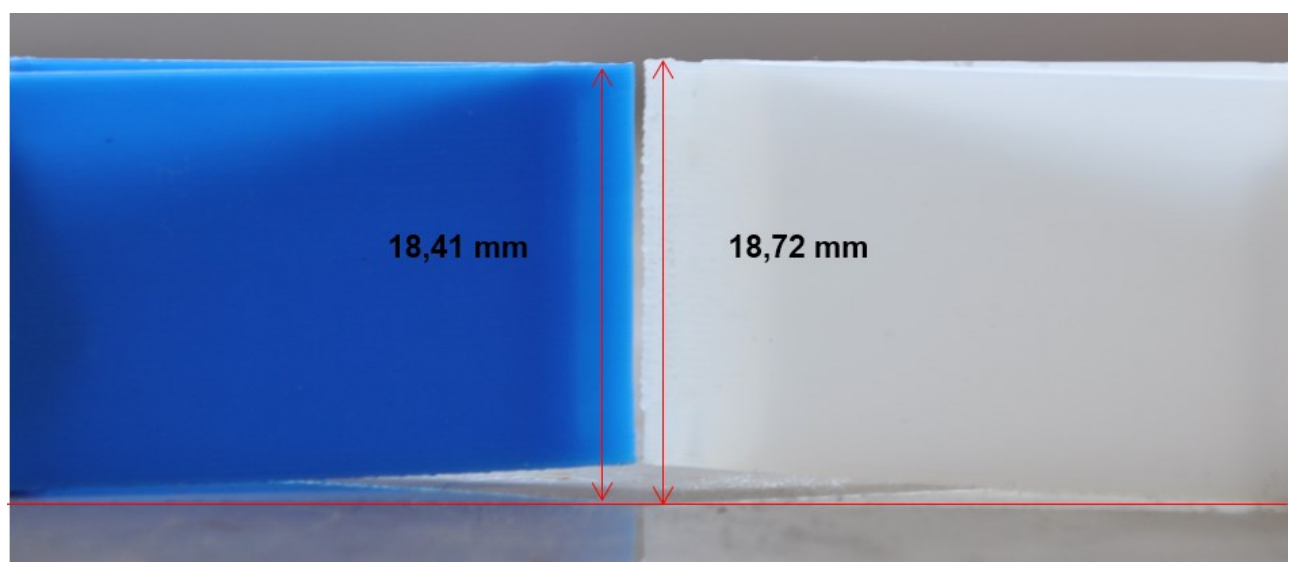

Fig. 4. View of the gripper finger base structure detachment from the work table- in the FDM printer (left) and the LPD one (right) 
The thickness of the individual section of the base structure work surface was measured, which is most likely to be destroyed during cyclic loading and the height of k (Fig. 5). For each dimension, there were 5 measurement attempts from which the maximum and minimum dimensions have been set and the average dimension was obtained from them.
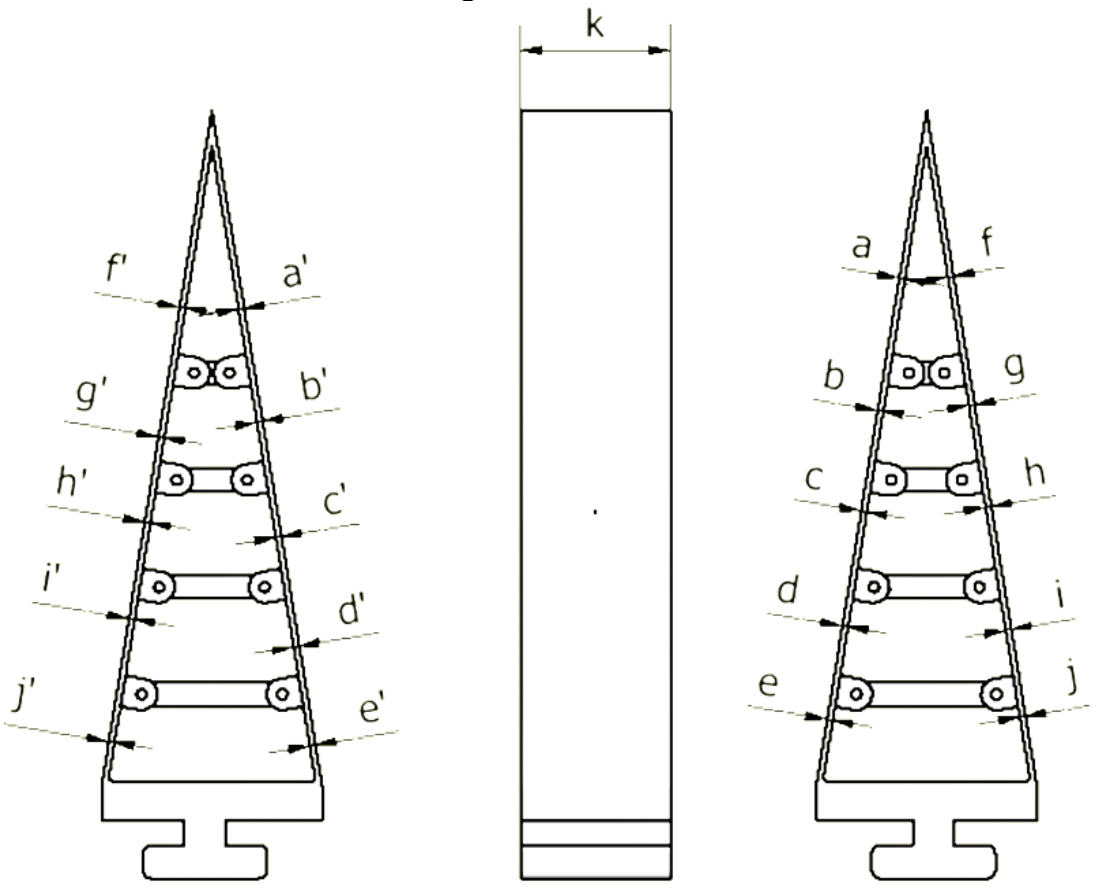

Fig. 5. Diagram of gripper finger with selected sections of the work surface of the base structure (a-j) and height $(\mathrm{k})$

On the basis of the measurements taken, the largest scatter for dimension $\mathrm{k}$, which is caused by the shrinking of the processed material from which the component is produced, may be noticed.

Table. 1 Results of measurements of a FDM specimen

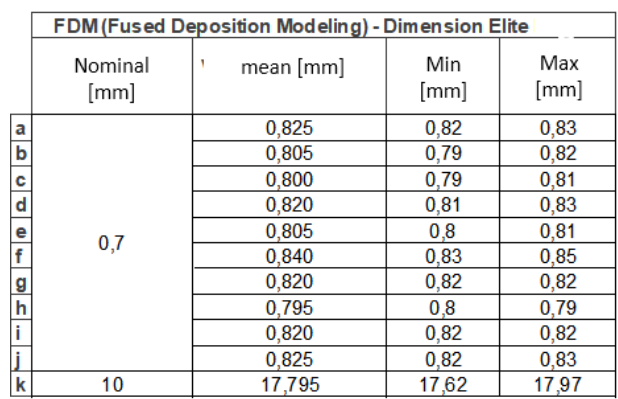

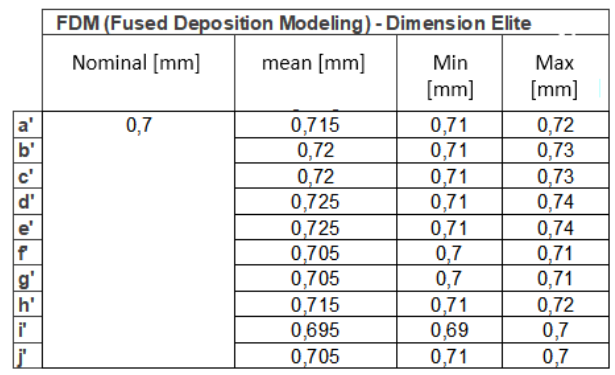

\section{Tests}

The fatigue test consisted of a cyclical, point-deflection of all three fabrication versions of gripper finger (Fig.6) on the test rig MTS 370.02 by Bionix. The amplitude and the deflection frequency are $10 \mathrm{~mm}$ and $2 \mathrm{~Hz}$ respectively. 


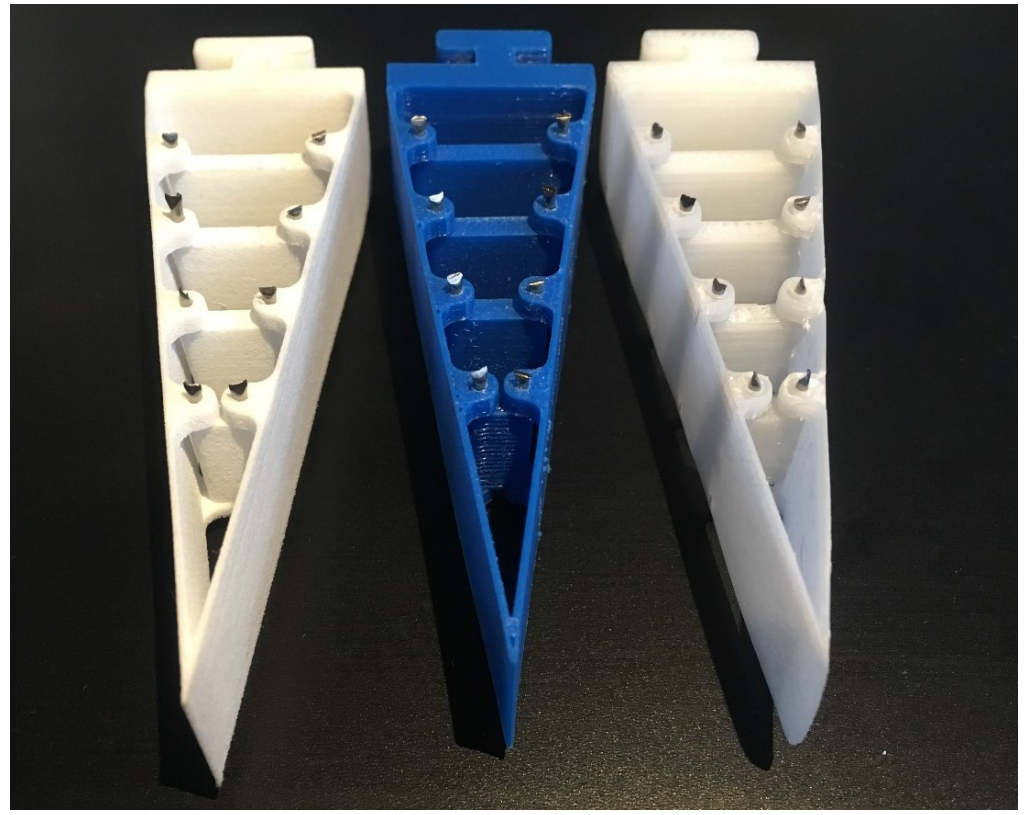

Fig. 6. Assembled gripper fingers printed in technologies (left to right): SLS, FDM, LDP

The previously designed and printed holder was mounted to the base of the load frame. Then the gripper finger was put in to the shape groove of the holder. A rounded mandrel has been mounted to the upper mounting of the load frame to transfer a specific point force to the tested component (Fig. 7).

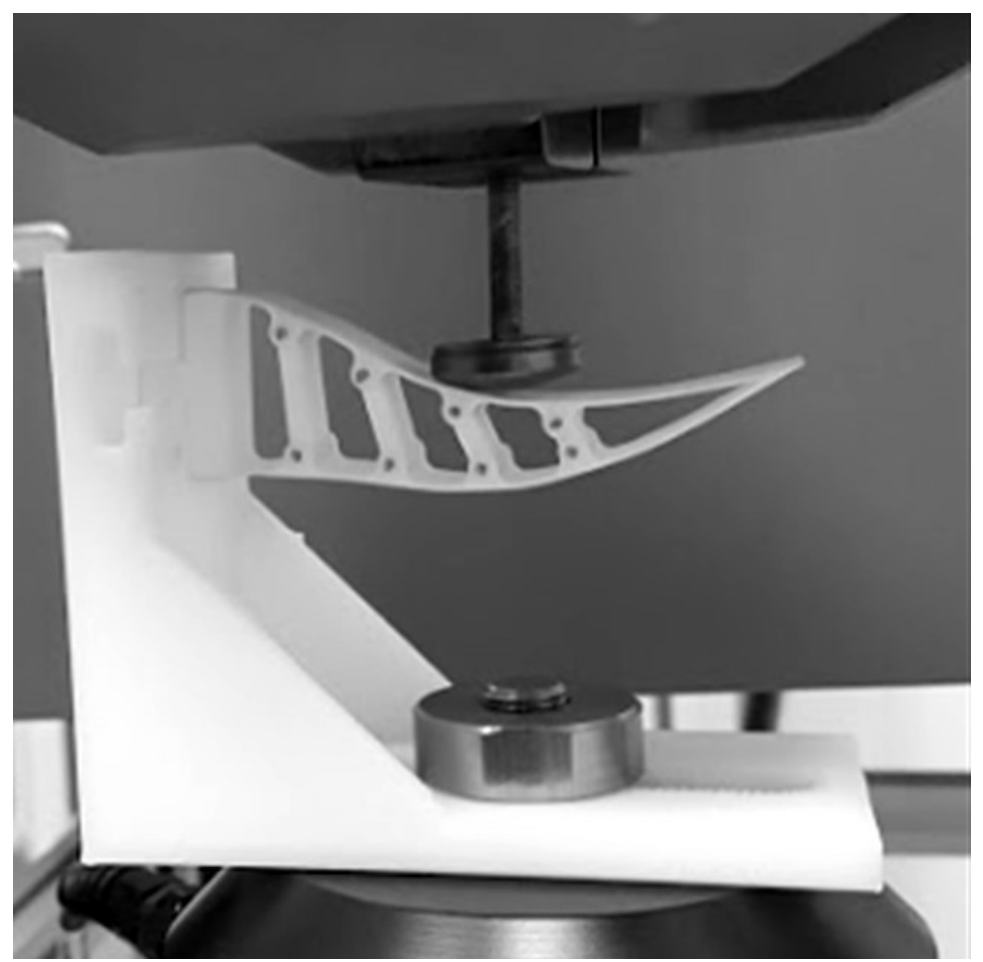

Fig. 7. One of the flexible fingers under load inside MTS 370.02 by Bionix 
Samples made on FDM and LDP printers were first tested and compared. The following effects can be observed from load diagram (Fig. 8):

- cycles 1-1110 (LPD sample) - Slow decrease of reaction force value under constant deflection - period of unstable work of the gripper finger,

- cycles 1100-1200 (LPD sample) - Rapid decrease in force value - permanent damage to the finger Gripper,

- cycle 1250 (LPD) - permanent damage of the gripper finger,

- cycles 1-950 (sample FDM) - Constant force value with constant deflection - stable working period of gripper finger,

- cycles 950-2200 (sample FDM) - Slow decrease in force value under constant deflection period of unstable operation of the gripper finger,

- cycle 2200 (sample FDM) - sudden decrease of a force value - the destruction of the gripper finger.

In the case of the LPD sample, the gripper's finger was destroyed at the point of the load of the mandrel (Fig. 9). The place of destruction shall coincide with the potential destruction area specified in the analysis carried out in Autodesk Inventor software. The safety factor here is only 1.09 .

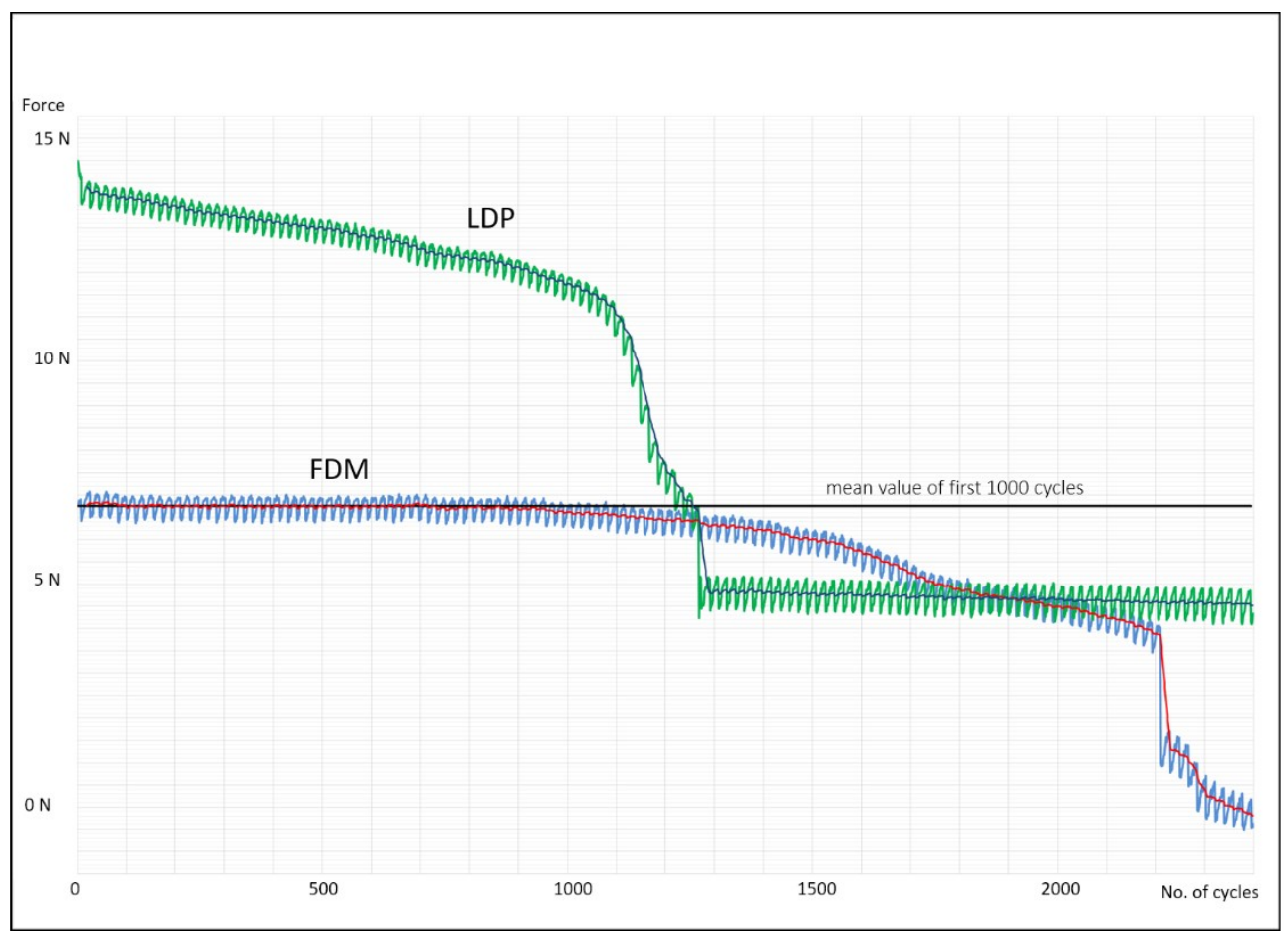

Fig. 8. Test results (reaction force vs number of deflection cycles) taken for LPD and FDM printed flexible fingers 


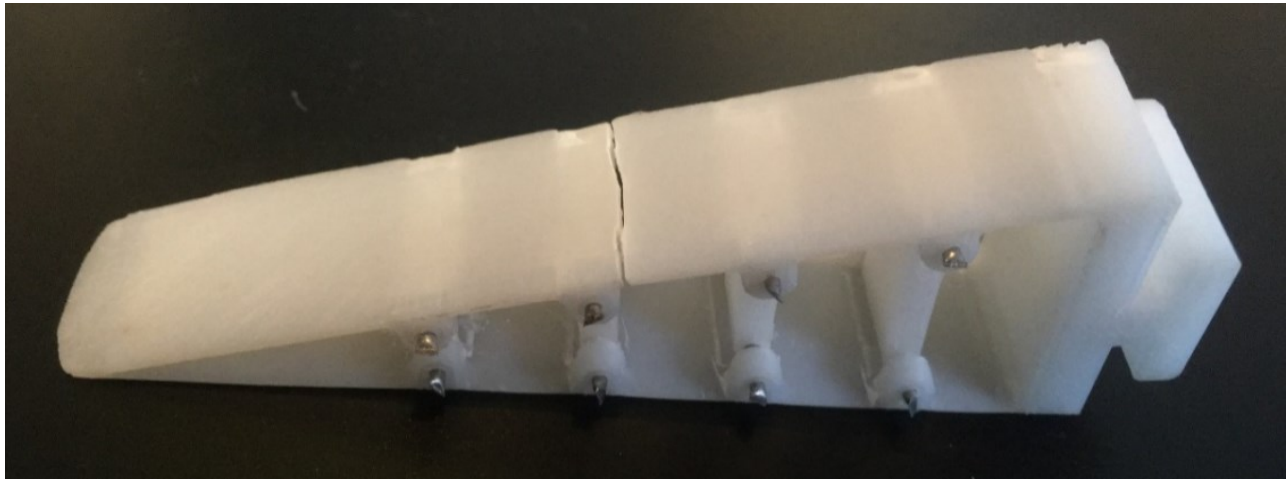

Fig. 9. View of a destroyed LPD sample after the fatigue test

The gripper finger made in FDM technology has been destroyed in the place shown in the following figure (Fig. 10). The location of the gripper's destruction also coincides with the potential damage area specified in Autodesk Inventor. The safety factor was 1.18. On the base structure of the gripper, there are traces of deformation under the action of a mandrel that generated load during the test.

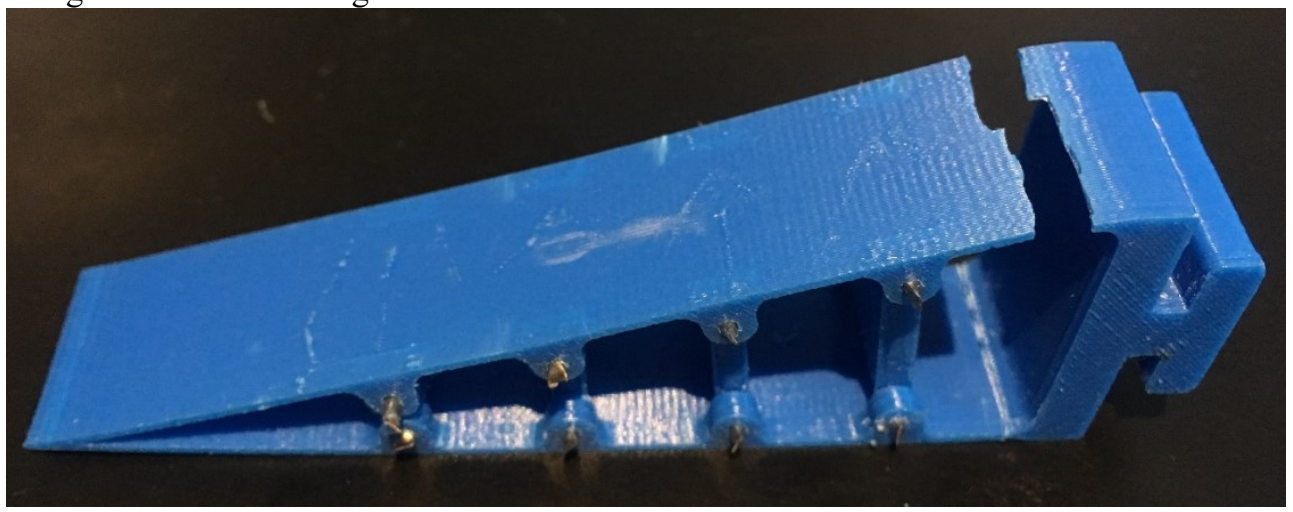

Fig. 10. View of a destroyed FDM sample after the fatigue test

The gripper made in SLS technology, similar to the original solution from FESTO, has been tested successively. The load chart (Fig. 11) analysis of the gripper fingers allows to identify the following phenomena:

- Cycles 1-10000 - constant value of the average load under the constant deformation value of the gripper's finger - the stable working range of the gripper finger,

- Cycles 10000-21600 - decrease the load value against deflection - slow but permanent increment of damaged material volume of the gripper's finger. 


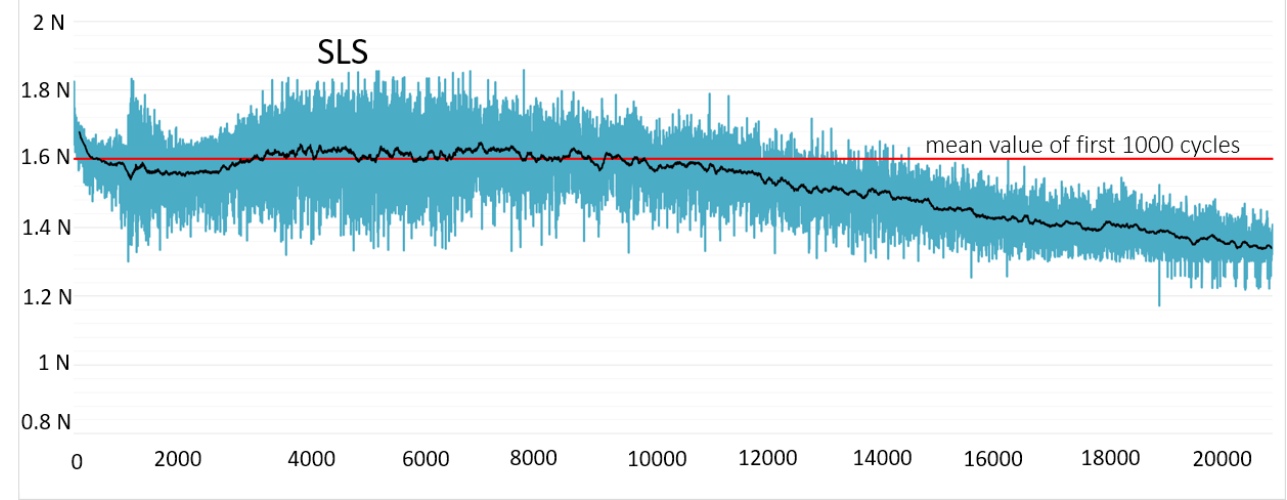

Fig. 11. Test results (reaction force vs number of deflection cycles) taken for SLS printed flexible finger

The sample made in SLS technique did not show any visible deformation or damage after 21600 cycles of its load (Fig. 12). It can be assumed that the decrease of reaction forces had an increasing effect along with the number of cycles and caused microcracks in the base structure of the gripper finger.

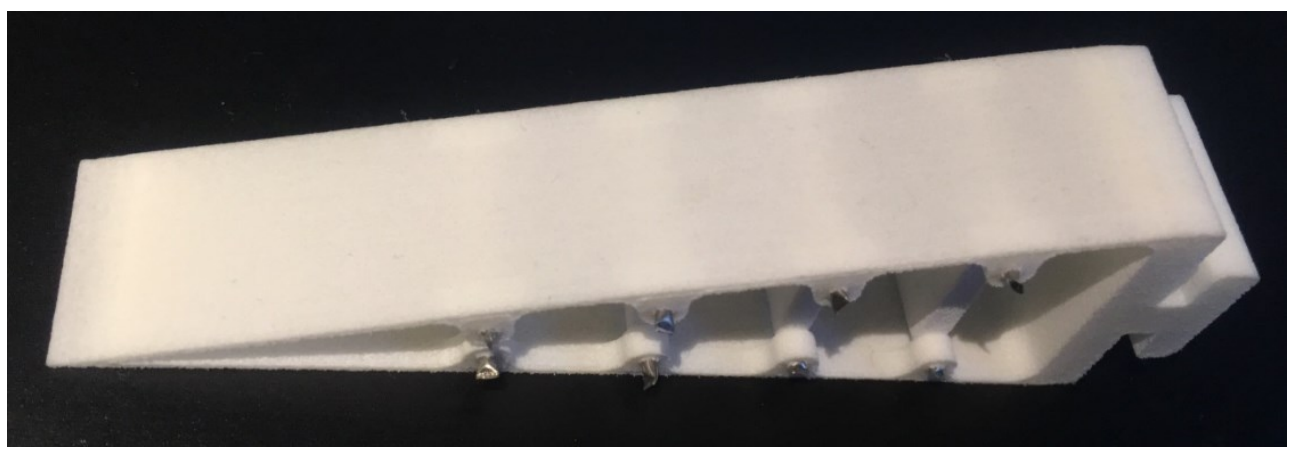

Fig. 12. View of a SLS sample after the fatigue test, without visible damages

\section{Conclusions}

The samples made in FDM technology are characterised by a stable operation in which the load/strain ratio remains relatively constant $\pm 0.5 \mathrm{~N}$, up to approx. 1000 cycles. Afterwards, a phase of unstable gripping operation took place, in which the gripper force changed from $6.5 \mathrm{~N}$ to $3.5 \mathrm{~N}$ in the 2200 cycle, after which the sample was permanently destroyed.

Samples made with LPD technology only allow for unstable operation up to approx. 1100 cycles, characterised by a change of force from $14.5 \mathrm{~N}$ to $11.5 \mathrm{~N}$. In subsequent cycles the load changes rapidly from $11.5 \mathrm{~N}$ to $6.5 \mathrm{~N}$ until the sample is destroyed around the cycle 1250 .

The elements made in SLS technique are characterised by the greatest possibilities of all three techniques tested in presented work. SLS printed finger has shown the possibility of stable operation of up to 10000 cycles but its subsequent use is characterised only by a slight and slow decrease of its stiffness.

Applications that enable load simulations can greatly help optimize the 3D CAD model before it is finished. The stress analysis made on the 3D CAD model in Autodesk Inventor has enabled the determination of areas of stress focus during the force. The location of the 
destruction of samples made by FDM and SLS technologies coincided with the most vulnerable areas to destruction in Autodesk Inventor software Stress Analysis

Prepared with a support of Statutory Found of Faculty of Mechatronics, Warsaw University of Technology

\section{References}

1. H. J. Warnecke, I. Schmidt, Flexible grippers for handling systems Design possibilities and experiences. International Journal of Production Research, 18(5), 525-537, (1980) DOI: $10.1080 / 00207548008919688$

2. Y. Zhang, F. Gao, Y. Zhang, W. A. Gruver, Dimensional synthesis of a flexible gripper with a high degree of stability. IEEE International Conference on Systems, Man and Cybernetics. Information Intelligence and Systems (Cat. No.96CH35929), 2, 1025-1030, Beijing, China, (1996) doi: 10.1109/ICSMC.1996.571221

3. P. Gorce, J.G. Fontaine, Design methodology approach for flexible grippers. Intell Robot Syst, 15(307), (1996) https://doi.org/10.1007/BF00572265

4. S. Zug, V. Müller, M. Seidel, P. Krenckel, Optimized configuration of a tactile sensor system for flexible grippers. 22nd IEEE International Conference on Emerging Technologies and Factory Automation (ETFA), 1-4, Limassol (2017) doi: 10.1109/ETFA.2017.8247763

5. Adaptive gripper, DHDG. FESTO, (2011/01)

6. R. Hagl, Das 3D-Druck-Kompendium Leitfaden für Unternehmer, Berater und Innovationstreiber (Springer Gabler Verlag) (2015)

7. http://bionictoys.de, (2018)

8. A. Fabio, Compliant Robot Gripper Won't Scramble Your Eggs, https://hackaday.com, (2013) 